dysfunction, and a gene locus mapped to chromosome 16. (Zorzi G, Conti C, Erba A et al. Paroxysmal dyskinesias in childhood. Pediatr Neurol March 2003;28:168-172). (Respond: Giovanna Zorzi MD, Department of Child Neurology, Instituto Nazionale Neurologico "C Besta", Milano, Italy).

COMMENT. Paroxysmal dyskinesias refer to brief attacks of dystonia or choreoathetosis, alone or combined, with return to normal between episodes. Cases are classified according to precipitating factors. Paroxysmal kinesthetic dyskinesia (PKD) is the most frequent form of paroxysmal dyskinesia, it is usually familial and idiopathic, often responds to treatment with carbamazepine, and may resolve spontaneously by age 30. PNKD is relatively rare, it is often symptomatic of illnesses including cerebral palsy, infection, or vascular lesion, and response to treatment is poor. Exercise-induced (PED) cases are rare, usually in response to running, and dystonia is generalized or focal, affecting the foot. Antiepileptic drugs, including carbamazepine, clonazepam, and acetazolamide, L-Dopa, and trihexyphenidyl are reported of benefit in some cases.

\title{
LANGUAGE DISORDERS
}

\section{LANGUAGE REGRESSION, AUTISM, AND EPILEPSY}

The records of 196 consecutive children (143 males and 53 females) with language regression evaluated between 1988 and 1994 by a child neurologist were analyzed at the Department of Neurology, Albert Einstein College of Medicine, Bronx, NY. Patients with a neurodegenerative or metabolic disorder, acquired brain injury, or Rett syndrome were excluded. Mean age at regression, defined as loss of previously acquired language skills, was 21.2 months (SD 10.5), the mean age at first visit was 50.6 months (SD 41.1), and the mean interval between onset and referral was delayed for 34.8 months (SD 38.3). Trigger factors that preceded the onset of language regression were reported in $74(38 \%)$; these were definite in $34(17 \%)$ and possibly related in $40(20 \%)$. They included illness $(23 \%)$, emotional upset $(16 \%)$, seizures $(3 \%)$, and trauma (1\%). A history of seizures was present in $15 \%$, but seizures were temporally related to language regression in only $3 \%$. Repeated ear infections were the most frequently reported abnormality in the past history, occurring in $32 \%$. At the time of neurologic examination, autistic spectrum disorder was diagnosed in $93 \%$, and cognitive impairment occurred in $73 \%$, with severe mental retardation in $3 \%$. Hearing was normal in $94 \%$, and mildly impaired in $6 \%$. Stereotypies were noted in $85 \%$, hypotonia in $58 \%$, toe walking in $15 \%$, and oromotor deficits in $11 \%$. Sensorimotor development was normal in $29 \%$. CT/MRI obtained in 75 children was abnormal in only 3 (4\%). Chromosomes and metabolic tests were normal in all 47 tested. EEGs showed paroxysmal abnormalities in 4 of $12(33 \%)$ with prolonged video monitoring. At follow-up (mean age 64 months) 39\% were nonverbal and an additional 45\% showed decreased verbal output. Some recovery occurred in $61 \%$ but only one child recovered fully. Improvement was most likely in the $49 \%$ who were developmentally normal before language regression was noted. (Wilson S, Djukic A, Shinnar S et al. Clinical characteristics of language regression in children. Dev Med Child Neurol August 2003;45:508-514). (Respond: Isabelle Rapin MD, Albert Einstein College of Medicine, K807, 1300 Morris Park Ave, Bronx, NY 10461). 
COMMENT. This study is similar to a multicenter study of language regression in a different cohort published earlier by the same investigators (Shinnar $\mathrm{S}$ et al. Pediatr Neurol 2001;24:183-189; see Ped Neur Briefs May 2001;15:37-38). As in the earlier publication that involved 177 children with early onset language regression, reports of a triggering event, frequently associated autistic symptoms, and the inordinately long delay in referral to a neurologist or language specialist are the most noteworthy findings. In patients with associated autism, one third show language regression between 18 and 24 months of age, and $95 \%$ before age 3 years, whereas in the majority of children with an associated epilepsy, as in Landau-Kleffner syndrome (LKS, acquired epileptic aphasia), regression begins after age 3 years (Deonna TW. J Clin Neurophysiol 1991;3:288-298). In a 10 year follow-up of children with LKS, children who showed language regression before 5 years of age were most likely to have severe language deficits (Bishop DVM. Dev Med Child Neurol 1985;27:705-712), and children with LKS or autism who were speaking at 5 years of age had a better prognosis.

The age-dependent characteristics of language regression are important in diagnosis and emphasize the need for early evaluation and treatment intervention. Children who show regression or a plateau in language development before 24 months of age should receive a neurologic examination that includes an electroencephalogram. EEG epileptiform discharges may occur in $16 \%$ of routine recordings and in $33 \%$ with prolonged video-EEG monitoring. Seizures temporally related to the onset of language regression should be treated with antiepileptic medication (AED). The response to conventional AEDs is variable (Paquier PF et al. Arch Neurol 1992;49:354-359), and a course of ACTH is more likely to result in remission of both seizures and aphasia. Treatment of LKS with ACTH or corticosteroids has been successful when given early; seizures and EEG epileptiform discharges were controlled within 3 weeks whereas complete remission of the acquired aphasia was delayed up to 8 months (Lerman P et al. Dev Med Child Neurol 1991;33:257-266). (See Progress in Pediatric Neurology II, PNB Publishers, 1994;pp223-226).

\section{INFECTIOUS DISORDERS}

\section{INFLUENZA-ASSOCIATED ENCEPHALOPATHY AND FEBRILE CONVULSIONS}

The transcription of cytokine genes in peripheral blood leukocytes (PBL) of 23 children (mean age 3.2 years) with influenza (mostly type A) complicated by encephalopathy (11 patients) or febrile convulsions (12 patients) was compared with systemic cytokine responses in 23 (mean age 5.0 years) with influenza but without neurologic complications, in a study at Nagoya University Graduate School of Medicine, Japan. WBCs were significantly higher in patients with encephalopathy than in patients with febrile convulsions or without neurologic complications $(\mathrm{P}=.02$ and $\mathrm{P}=.002$, respectively). A quantitative polymerase chain reaction (PCR) showed that transcription of the interleukin (IL)-6, IL-10, and tumor necrosis factor (TNF)-a genes was up-regulated more in patients with encephalopathy than in those without $(\mathrm{P}=.049, \mathrm{P}=.049$, and $\mathrm{P}=.098$, respectively). Of 3 patients with encephalopathy and extremely high concentrations of IL10, 2 died. IL-10 and TNF-a transcriptions were also higher in patients with febrile 